\title{
A influência das redes de alianças estratégicas sobre a tecnologia de informação e comunicação*
}

\author{
Marcos Vianna Villas** \\ T. Diana L. v. A. de Macedo-Soares***
}

\begin{abstract}
SumÁRIo: 1. Introdução; 2. Redes de alianças estratégicas (RAEs); 3. Tecnologia de informação de comunicação (TIC); 4. A influência das RAEs sobre a TIC da empresa focal; 5. Metodologia de pesquisa; 6. Resultados; 7. Discussão; 8. Conclusão.
\end{abstract}

Summary: 1. Introduction; 2. Strategic alliance networks (SANs); 3. Information and communication technology (ITC); 4. The influence of SANs on the focal firm's ITC; 5. Research method; 6. Results; 7. Discussion; 8. Conclusion.

Palavras-chave: rede de alianças estratégicas; tecnologia de informação e comunicação; gestão.

KEY worDs: strategic alliance network; information and communication technology; management.

As empresas cada vez mais se relacionam estrategicamente com outras empresas, com vistas a terem maior competitividade. Um dos principais fatores organizacionais internos de apoio à estratégia de uma empresa é a tecnologia de informação e comunicação (TIC). Este artigo apresenta os resultados de uma investigação empírica das percepções de gestores de TIC com atuação no Brasil. Foram investigadas as influências que 13 características de uma rede de alianças estratégicas (RAE) têm sobre quatro propriedades da TIC de uma empresa: maturidade, complexidade, flexibilidade e alinhamento. Os resultados da pesquisa exploratória sugerem que essa

\footnotetext{
* Artigo recebido em set. 2007 e aceito em abr. 2008. Este artigo foi apresentado no Congresso Internacional, Gbata 2007, em Taipei, Taiwan, onde ganhou o "Best Paper Award", e foi publicado no JGBAT, Fall 2007.

** DSc pela Pontifícia Universidade Católica do Rio de Janeiro (PUC-Rio). Professor adjunto da PUC-Rio/Departamento de Informática (DI). Endereço: Av. Marquês de São Vicente, 225 — Gávea — CEP 22453-900, Rio de Janeiro, RJ, Brasil. E-mail: villas@inf.puc-rio.br.

*** PhD pela Pontifícia Universidade Católica do Rio de Janeiro (PUC-Rio). Professor associado da PUC-Rio/Departamento de Administração (IAG). Endereço: Av. Marquês de São Vicente, 225 — Gávea — CEP 22453-900, Rio de Janeiro, RJ, Brasil. E-mail: redes@strategy-research.com.br.
} 
influência existe e que se manifesta de várias formas e com distintos graus de força. Há evidências que a maturidade — grau de evolução ou competência — de TIC é a mais fortemente afetada pelas 13 características de RAE investigadas e que a equivalência estrutural é aquela que menos influencia a TIC de uma empresa. Inferimos que, uma vez compreendida as possíveis influências de uma RAE, os gestores de TIC podem antecipar-se a estas influências e planejar melhor a TIC das suas empresas para dar sustentação à sua competitividade.

\section{The influence of strategic alliance networks on information and communication technology}

More and more firms are establishing strategic relationships with other firms in order to enhance their competitiveness. One of the main internal organizational factors used to support this strategy is information and communication technology (ICT). This article shares the main results of an empirical investigation into the perceptions of ICT managers who operate in Brazil. The investigation analyzed the influences of 13 characteristics of a strategic alliance network (SAN) on four properties of a firm's ICT: maturity, complexity, flexibility, and alignment. Its results suggest that this influence exists and that it manifests itself in various ways, and with different degrees of strength. There is evidence that ICT maturity — degree of development or competence - is the property most strongly affected by the 13 SAN characteristics and that structural equivalence is the one that less influences ICT. The authors infer that, once the possible influences of a SAN have been understood, ICT managers can anticipate these influences and better plan their firms' ICT in order to sustain their competitiveness

\section{Introdução}

Nos ambientes de negócios das empresas, de várias indústrias no mundo, há poucos fatos e leis imutáveis e muitas incertezas e desafios. As mudanças são constantes e cada vez mais aceleradas. As empresas necessitam adequar-se às novas situações rápida e continuamente, pela redefinição e adaptação de suas estratégias (Rockart, Earl e Ross, 1996). Da mesma forma, a estratégia de tecnologia de informação e comunicação (TIC) precisa estar sempre coerente (Villas, Macedo-Soares e Fonseca, 2006) e, portanto, ser realinhada quando necessário, dinamicamente, à estratégia da empresa de modo a acompanhar suas necessidades (Prahalad e Krishnan, 2002).

Um dos efeitos da acirrada competição entre as empresas é a especialização. Cada vez mais as empresas buscam concentrar suas atividades no que elas têm de maior valor e que seja único para seus clientes, ou seja, nas suas competências essenciais (Prahalad e Hamel, 1990). Isso explica em grande 
parte porque elas formam redes de relacionamentos com outras empresas, notadamente alianças estratégicas de várias naturezas e intensidades (por exemplo, acordos, contratos e joint ventures), de modo a complementar suas competências com as das outras empresas parceiras para poder atender melhor aos seus clientes.

A TIC tem papel relevante nessas redes de alianças estratégicas (RAEs). Quanto mais intenso o relacionamento entre uma empresa e sua RAE, maior será a comunicação e troca de informação entre elas (Lia et al., 2006; Rockart, 1998; Sampler e Short, 1998; Silva e Alcântara, 2001). Da mesma forma que a TIC melhora a comunicação interna entre as áreas de uma empresa, ela também traz mais velocidade e confiabilidade à troca de informações entre as empresas de uma RAE. Processos de negócio podem passar a ser interempresas e não apenas intra-empresa. Por outro lado, relacionamentos intensos podem prender as empresas envolvidas (lock-in), impedindo que elas estabeleçam relacionamentos com outros parceiros mais interessantes.

Há duas certezas, portanto: para estarem aptas a competir, cada vez mais as empresas precisam tanto estar relacionadas estrategicamente com outras empresas em RAEs, quanto ter recursos de TIC coerentes com a sua estratégia e adequados a essa realidade.

Este artigo investiga as influências que a RAE de uma empresa tem sobre os recursos organizacionais relacionados à TIC dessa mesma empresa. De acordo com a revisão da literatura, não foram realizados estudos voltados especificamente para a análise do impacto da RAE sobre a TIC de uma empresa focal. Aqui são apresentados os principais resultados de uma investigação empírica, conduzida no Brasil pelos autores, que buscou preencher essa lacuna.

$\mathrm{O}$ artigo está dividido em seis partes. Na primeira, apresentamos o referencial teórico de pesquisa pertinente a RAEs, TIC e a influência de RAEs sobre a TIC de uma empresa. Na segunda, descrevemos os métodos adotados na investigação e, na terceira parte, compartilhamos seus principais resultados. Na quarta, estes são discutidos e na quinta parte, após uma avaliação geral da contribuição da pesquisa, são identificadas as influências mais críticas de características das RAEs sobre as propriedades de TIC de uma empresa. Na última parte, formulamos algumas considerações finais.

\section{Redes de alianças estratégicas (RAEs)}

A estratégia de negócios de uma empresa tem por objetivo assegurar sua existência e lucratividade. A estratégia define a identidade e os objetivos da 
empresa, orientando, portanto, suas ações. O processo de planejamento estratégico é contínuo e suas revisões cada vez mais freqüentes, em função da velocidade das mudanças do cenário competitivo (macroambiente) em uma determinada indústria.

Em complementação à análise dita "tradicional", notadamente da visão baseada em recursos (RBV), Dyer e Singh (1998) sugerem que os recursos estratégicos de uma empresa também podem estar localizados nos relacionamentos que essa empresa mantém com outras empresas. Assim, o nível de análise da competitividade de uma empresa na sua indústria não pode estar centrado apenas nas características dessa empresa e de seu meio, mas também deve considerar as características dos relacionamentos e das redes por eles constituídos - deve envolver uma análise relacional. Esses autores consideram que a vantagem competitiva de uma empresa pode estar, em termos de sua rede, nos recursos (pessoas, equipamentos, processos), no conhecimento (a interação que possibilita transferência, recombinação e inovação), na complementaridade de recursos e competências (onde o resultado é maior que a soma das partes) e na efetiva governança (para reduzir custos de transação), específicos deste relacionamento. Neste caso, haveria um lucro acima do normal gerado pela relação entre as empresas, pelas contribuições complementares e peculiares de cada uma delas, de forma que este lucro não poderia ser gerado por nenhuma das empresas envolvidas isoladamente.

Entre esses relacionamentos, os que são objeto do estudo em questão neste artigo são as redes de alianças estratégicas: conjunto de relacionamentos duradouros, de significado estratégico para as partes, onde são considerados todos os possíveis tipos de relacionamento entre os diversos atores de uma indústria, entre indústrias e entre países (Gulati, Nohria e Zaheer, 2000). Ainda segundo Gulati (1998) as alianças estratégicas são, entre as redes estratégicas, os arranjos formados voluntariamente entre empresas. Macedo-Soares (2002) complementa essa definição, afirmando que as alianças são estratégicas quando contribuem diretamente para a vantagem competitiva de uma empresa.

Do ponto de vista de uma empresa em particular, denominada "empresa focal", existe uma sub-rede dos relacionamentos estratégicos nos quais a empresa participa mais diretamente, denominada "ego-rede" (Macedo-Soares, 2002). Uma ego-rede é formada pela empresa focal (ego), as organizações a ela diretamente relacionadas (alters) e todos os relacionamentos existentes entre elas (ego-alter e alter-alter). Uma empresa deseja que sua ego-rede sempre lhe seja benéfica de modo que, ao associar-se com outras organizações, seus objetivos estratégicos sejam mais facilmente atingidos e sua vantagem competitiva seja sustentável. Essa rede passa a ter valor estratégico para a 
"empresa focal" ao melhorar, entre outros fatores, seu posicionamento estratégico (Porter, 1979), aumentando assim o seu poder de barganha na indústria, por exemplo, pela maior escala de compras, ao mesmo tempo em que pode criar barreiras para entrada de novos rivais, pelo uso de tecnologias inovadoras e proprietárias de parceiros ou pela criação de plataforma ou padrões tecnológicos que reforcem esses relacionamentos (Shapiro e Varian, 1999).

Macedo-Soares (2002) montou listas de referência para o seu modelo de análise estratégica no caso de empresas em alianças e redes (SNA - strategic network analysis), com variáveis e constructos tanto no nível da empresa quanto no nível da indústria, para captar dados sobre as implicações estratégicas das características de uma rede de relacionamentos nesses níveis. Baseouse para tanto nos trabalhos de Galaskiewicz e Zaheer (1999), Gulati, Nohria e Zaheer (2000), Kale, Singh e Perlmutter (2000) e Knoke (2001). Das listas de referência propostas foram escolhidas as seguintes características de RAE para investigação na presente pesquisa, apresentadas no quadro 1 .

\section{Quadro 1 \\ Características de RAE}

\begin{tabular}{|c|c|}
\hline Característica de RAE & Definição \\
\hline Cardinalidade & Quantidade de membros que compõem a rede de alianças estratégicas. \\
\hline Densidade & $\begin{array}{l}\text { Proporção de laços observados em relação ao número de conexões } \\
\text { possíveis. Uma rede com densidade máxima é aquela onde há laços } \\
\text { entre todos os membros da rede. }\end{array}$ \\
\hline Centralidade & $\begin{array}{l}\text { Quantidade de laços da empresa focal com os demais membros da } \\
\text { rede, relativa à quantidade de laços dos demais membros da rede. }\end{array}$ \\
\hline Equivalência estrutural & $\begin{array}{l}\text { Extensão com que os membros da rede têm laços com características } \\
\text { semelhantes com outros membros, por exemplo, ao compartilhar os } \\
\text { mesmos clientes. }\end{array}$ \\
\hline Status da empresa focal & $\begin{array}{l}\text { Importância da empresa focal na rede, determinada pelo seu tamanho, } \\
\text { papel e/ou recursos. Um bom indicador de status, quando aplicável, é a } \\
\text { sua posição em rankings consagrados como Exame Maiores e Melhores } \\
\text { e Valor Econômico, entre outros. }\end{array}$ \\
\hline Status dos parceiros & $\begin{array}{l}\text { Importância dos outros membros (todos exceto a empresa focal) } \\
\text { na rede, determinada pelo seu tamanho, papel e/ou recursos. Um } \\
\text { bom indicador de status, quando aplicável, é a posição dos parceiros } \\
\text { em rankings consagrados como Exame Maiores e Melhores e Valor } \\
\text { Econômico, entre outros. }\end{array}$ \\
\hline $\begin{array}{l}\text { Natureza dos } \\
\text { relacionamentos }\end{array}$ & $\begin{array}{l}\text { Considera que o relacionamento estratégico entre cada par de membros } \\
\text { pode ser oportunista (ganha-perde) ou colaborativo (ganha-ganha). }\end{array}$ \\
\hline $\begin{array}{l}\text { Força dos } \\
\text { relacionamentos }\end{array}$ & $\begin{array}{l}\text { Considera que cada par de membros em um relacionamento estratégico } \\
\text { tem o seu grau de comprometimento com esse relacionamento. Pode } \\
\text { ser caracterizada pelo tipo de aliança estratégica e pela freqüência de } \\
\text { interações. }\end{array}$ \\
\hline
\end{tabular}




\begin{tabular}{|c|c|}
\hline Característica de RAE & Definição \\
\hline $\begin{array}{l}\text { Experiência com alianças } \\
\text { estratégicas }\end{array}$ & $\begin{array}{l}\text { Experiência da empresa focal, caracterizada pela duração de alianças } \\
\text { estratégicas formadas e pela quantidade total de parceiros estratégicos. }\end{array}$ \\
\hline $\begin{array}{l}\text { Volume de investimentos } \\
\text { específicos }\end{array}$ & $\begin{array}{l}\text { Volume de investimentos realizados pela empresa focal especificamente } \\
\text { nas suas alianças estratégicas. }\end{array}$ \\
\hline $\begin{array}{l}\text { Mecanismo de } \\
\text { governança }\end{array}$ & $\begin{array}{l}\text { O mecanismo de governança de uma rede é um conjunto de } \\
\text { salvaguardas dos relacionamentos entre as partes, de modo que } \\
\text { nenhuma delas perceba benefícios em comportamentos oportunistas. }\end{array}$ \\
\hline $\begin{array}{l}\text { Grau de gestão de } \\
\text { mudanças na rede }\end{array}$ & $\begin{array}{l}\text { A gestão de mudanças das alianças estratégicas na rede é determinada } \\
\text { pela qualidade dos processos dessa gestão. }\end{array}$ \\
\hline $\begin{array}{l}\text { Grau de alinhamento } \\
\text { dinâmico múltiplo }\end{array}$ & $\begin{array}{l}\text { Desenvolvimento de competências e processos que garantam e sus- } \\
\text { tentem a compatibilidade entre os parceiros quanto à estratégia, estilos } \\
\text { gerenciais, cultura e complementaridade de recursos. }\end{array}$ \\
\hline
\end{tabular}

\section{Tecnologia de informação de comunicação (TIC)}

As empresas, para serem competitivas, utilizam e dependem da infra-estrutura tecnológica composta de computadores, programas, sistemas de informação e meios de comunicação entre as partes (ex.: redes de computadores), as quais, por sua vez, dependem de pessoas e processos adequados para sua manutenção e evolução (Marchand, Kettinger e Rollins, 2000). As TICs, ou seja, tecnologias de informática e de telecomunicações aplicadas ao processamento, armazenamento e transmissão de informação em formato digital (Carr, 2003), trazem mais velocidade e confiabilidade, entre outros, à troca de informações entre as diversas áreas de uma empresa como, por exemplo, na organização das atividades das empresas em torno dos seus processos (Hammer e Champy, 1995).

O termo TIC é recente e vem substituindo o termo TI (tecnologia de informação), que por sua vez substituiu o termo informática no que se refere às tecnologias acima citadas, aplicadas no contexto das organizações. Com a crescente importância para as empresas da comunicação entre computadores (Andersen, 2001), inicialmente com o electronic data interchange - EDI (troca eletrônica de dados) e mais recentemente com o uso comercial da internet, a dimensão de comunicação dessas tecnologias passou a ser enfatizada. Termos como telemática e teleinformática, menos utilizados, também enfatizam a convergência entre as tecnologias que formam as TICs. Embora muitos autores não explicitem essa dimensão nos seus textos, consideramos que referências à TI são referências à TIC.

A função de TIC em uma empresa possui diversos elementos. Para o Cobit - Control objectives for information and related technology - (ITGI, 2007), 
framework orientado para gestão e governança da TI em uma empresa, a arquitetura de TI de uma empresa é formada pelos seus processos de TI e quatro recursos: aplicações, infra-estrutura, informação e pessoas. Consideramos neste artigo que a função de TIC em uma empresa possui os seguintes elementos:

v infra-estrutura - instalações, computadores (servidores, estações de trabalho, mainframes), redes de computadores (rede lógica, acesso à internet, firewall etc.), programas básicos (sistemas operacionais, sistemas gerenciadores de banco de dados etc.) e de produtividade (processador de texto, planilha eletrônica, correio eletrônico etc.) é equivalente ao recurso "infraestrutura" do Cobit (ITGI, 2007);

v aplicações — sistemas de informação que apóiam os processos de negócio da empresa, incluindo os IOS - sistemas interorganizacionais —, inclui os recursos "aplicações" e "informações" do Cobit (ITGI, 2007);

v processos - atividades de atendimento aos usuários, aquisição, suporte, manutenção, acompanhamento e gestão da infra-estrutura e desenvolvimento, aquisição e manutenção de aplicações, inclui os processos de TIC relacionados aos domínios "adquirir e implementar", "entregar e suportar" e "monitorar e avaliar" do Cobit (ITGI, 2007);

v equipe - recursos humanos, que executam os processos da função de TIC, e a sua organização, em equipes, papéis e responsabilidades, inclui o recurso "pessoas" do Cobit (ITGI, 2007);

- gestão - responsável por processos como planejamento estratégico de TIC e gerenciamento de orçamentos, despesas e equipe de TIC, inclui os processos de TIC relacionados ao domínio "planejar e organizar" do Cobit (ITGI, 2007).

A função de TIC em uma empresa possui diversas características e propriedades. Uma das propriedades mais pesquisadas é o seu alinhamento à estratégia de negócios da empresa (Henderson e Venkatraman, 1993; Broadbent e Weill, 1997; Luftman, Papp e Brier, 1999). Considerando-se que o alinhamento deva ser dinâmico, em especial nas empresas que têm suas atividades em ambientes turbulentos, alguns autores pensam que é necessária maior flexibilidade de TIC (Knoll e Jarvenpaa, 1994; Byrd, 2001; Prahalad e Krishnan, 2002). Soluções flexíveis têm custos e riscos associados a maiores níveis de complexidade em uma organização (Duimering, Safayeni e Purdy, 1993). Mais recentemente, as organizações têm buscado avaliar a maturidade da função de TIC de modo a identificar qual o seu desempenho, como ele é 
comparado ao das outras organizações na mesma indústria e qual a meta para melhoria (ITGI, 2007).

As propriedades de TIC que foram investigadas neste artigo são: maturidade - grau de evolução ou competência dos elementos de TIC; complexidade - medida em que cada elemento de TIC possui subelementos e variações, necessárias às suas atividades; flexibilidade - capacidade de reação dos elementos de TIC a novas necessidades da empresa; e alinhamento - grau de coerência e convergência dos elementos de TIC à estratégia de negócios da empresa.

\section{A influência das RAEs sobre a TIC da empresa focal}

O estudo do impacto de TIC no caso de empresas que atuam em RAEs é recente. Uma das primeiras referências é o trabalho de McFarlan (1984), que observa que os sistemas de informação que operam em uma rede interorganizacional podem alterar, de forma estratégica, o poder de barganha entre empresas compradoras e fornecedoras. Já na década de 1990, McFarlan (1990) apresentou alguns tipos de "parcerias de informação", principalmente em atividades de marketing, e seus fatores de sucesso. Segundo seu estudo, as empresas que desenvolvessem relacionamentos colaborativos de TIC poderiam utilizar novos canais de distribuição e obter eficiências operacionais (menor custo de transação). Os fatores de sucesso identificados eram: a visão compartilhada na alta administração; habilidades complementares em TIC, cujo resultado é semelhante ao que foi encontrado por Harrigan (1998, citado por Kale, Singh e Perlmutter, 2000) para o constructo adequação de parceiros (partner fit) ao avaliar a complementaridade entre eles, na dimensão de análise relacional no nível da empresa denominada "administração da rede" (Gulati, Nohria e Zaheer, 2000; Macedo-Soares e 2002; Macedo-Soares; Lange, 2004); planejamento para sucesso em curto prazo; perseverança na qualidade das informações; e arquitetura de negócios apropriada, entre parceiros.

Os sistemas de informação segundo Konsynski (1993), ou de forma mais abrangente TIC em geral (Rockart, 1998), têm papel fundamental na "extensão" de uma empresa para além das suas fronteiras organizacionais e na modificação das relações interorganizacionais, da competitividade de uma indústria e da prática de cooperação. A empresa deixa de ter uma fronteira bem definida para se confundir com a sua RAE. Konsynski (1993) afirma que quanto mais uma empresa tem controle sobre o "sistema de informações interorganizacional", maior será seu entendimento do processo e maior será o seu 
poder de influência sobre seus parceiros, em especial no acesso à informação. A TIC também teria papel de construir e manter parcerias sustentáveis.

Dewett e Jones (2001) afirmam que TIC, da mesma forma que traz eficiência no relacionamento entre as áreas de uma empresa, influencia os relacionamentos interorganizacionais, em especial o custo de transação, pois informações podem ser transmitidas - por meio de redes de computadores - e utilizadas a baixo custo. Toda uma cadeia de valor, de fornecedores a clientes, pode ser integrada por uma rede de computadores, diminuindo custos e criando barreiras de saída. A TIC tem sido utilizada cada vez mais em alianças estratégicas, rompendo barreiras entre indústrias e integrando diferentes cadeias de valor. De fato, a TIC potencializa a colaboração entre especialistas de empresas estrategicamente aliadas, o que é um fator de inovação.

Para que existam processos interempresas, é necessário que fatores organizacionais como a infra-estrutura, os processos de TIC e as pessoas neles envolvidas estejam operando de forma coordenada, de modo que as informações possam fluir, de forma rápida, automática e controlada, entre as empresas. O problema está no fato de que esses fatores nas diferentes empresas participantes de uma RAE nem sempre são congruentes, pois cada uma dessas empresas tem seus próprios interesses, sua estratégia de negócios e, conseqüentemente, sua estratégia de TIC.

\section{Metodologia de pesquisa}

A pesquisa foi essencialmente empírica e exploratória (Remenyi et al., 1998). O universo considerado foi o de gestores de TIC experientes, com atuação no Brasil, cuja experiência tenha sido em empresas que utilizam TIC de forma significativa (não apenas como suporte à comunicação entre pessoas, como por exemplo pelo uso de correio eletrônico). Os sujeitos foram os próprios gestores de TIC.

Para a pesquisa telematizada foi realizada uma amostragem não-probabilística do tipo "bola-de-neve", devido à dificuldade de identificação dos sujeitos (Rea e Parker, 2000:150). A revisão de literatura, com vistas à construção do referencial teórico da presente pesquisa, seguiu a metodologia proposta por Russo, Villas e Macedo-Soares (2006).

O meio de coleta de dados foi um questionário telematizado adaptativo (website). As perguntas foram agrupadas em blocos para caracterização do respondente (B1), caracterização da empresa onde trabalha (B2), caracterização das alianças estratégicas e da RAE da empresa onde trabalha (B3), e 
registro da percepção do respondente relativa à influência de características de RAEs sobre quatro propriedades da TIC da empresa focal (B4). Caso a empresa onde estivesse empregado o respondente não possuísse alianças estratégicas, o bloco B3 não era apresentado; caso o respondente não estivesse empregado como funcionário de alguma empresa, os blocos B2 e B3 não eram apresentados. O quinto e último bloco (B5) buscou identificar outros possíveis respondentes para o questionário ("bola-de-neve").

De modo a forçar um posicionamento a respeito da influência das características de RAE sobre cada propriedade da TIC da empresa focal, foi definida uma escala dicotômica para cada uma dessas características, com um valor negativo (-) e outro positivo (+), bem como uma orientação para definição da característica, apresentada no quadro 2 . Uma escala dicotômica não permite a avaliação das intensidades das influências, porém foi considerada adequada para a presente pesquisa, que tem por objetivo descobrir a existência (ou não) de influências.

Quadro 2

Escala dicotômica das características de RAE

\begin{tabular}{|c|c|c|c|}
\hline $\begin{array}{l}\text { Característica } \\
\text { de RAE }\end{array}$ & $\begin{array}{c}\text { Valor negativo } \\
(-)\end{array}$ & $\begin{array}{c}\text { Valor positivo } \\
(+)\end{array}$ & Orientação \\
\hline Cardinalidade & Baixa & Alta & $\begin{array}{l}\text { Uma rede será considerada de alta } \\
\text { cardinalidade quando ela tiver nove ou mais } \\
\text { membros no total. }\end{array}$ \\
\hline Densidade & Baixa & Alta & $\begin{array}{l}\text { Uma rede será considerada de alta densidade } \\
\text { quando esta for acima de } 60 \% \text { com relação } \\
\text { ao máximo possível de laços. }\end{array}$ \\
\hline Centralidade & Não é central & É central & $\begin{array}{l}\text { Uma empresa focal é dita central quando, na } \\
\text { sua ego-rede, ela possui mais laços com os } \\
\text { outros membros desta rede do que qualquer } \\
\text { outro membro desta mesma rede. }\end{array}$ \\
\hline $\begin{array}{l}\text { Equivalência } \\
\text { estrutural }\end{array}$ & $\begin{array}{l}\text { Não há } \\
\text { equivalência } \\
\text { estrutural }\end{array}$ & $\begin{array}{l}\text { Há equivalência } \\
\text { estrutural }\end{array}$ & $\begin{array}{l}\text { Há equivalência estrutural se membros } \\
\text { da rede têm laços com características } \\
\text { semelhantes com outros membros, por } \\
\text { exemplo, ao compartilhar os mesmos clientes. }\end{array}$ \\
\hline $\begin{array}{l}\text { Status da } \\
\text { empresa focal }\end{array}$ & Baixo & Alto & $\begin{array}{l}\text { Se a empresa focal tem o maior tamanho } \\
\text { entre os membros da rede, e é a principal } \\
\text { cliente ou fornecedora de seus parceiros } \\
\text { estratégicos, seu status é alto. }\end{array}$ \\
\hline
\end{tabular}

Continua 


\begin{tabular}{|c|c|c|c|}
\hline $\begin{array}{l}\text { Característica } \\
\text { de RAE }\end{array}$ & $\begin{array}{c}\text { Valor negativo } \\
(-)\end{array}$ & $\begin{array}{c}\text { Valor positivo } \\
(+)\end{array}$ & Orientação \\
\hline $\begin{array}{l}\text { Status dos } \\
\text { parceiros }\end{array}$ & Baixo & Alto & $\begin{array}{l}\text { Se os outros membros são maiores que a } \\
\text { empresa focal, e são seus principais clientes } \\
\text { ou fornecedores, o status dos parceiros é } \\
\text { alto. }\end{array}$ \\
\hline $\begin{array}{l}\text { Natureza dos } \\
\text { relacionamentos }\end{array}$ & Oportunista & Colaborativa & $\begin{array}{l}\text { Se a rede tem relacionamentos } \\
\text { predominantemente colaborativos (ganha- } \\
\text { ganha), ela é dita colaborativa; caso contrário } \\
\text { (predominância de laços ganha-perde) ela é } \\
\text { dita oportunista. }\end{array}$ \\
\hline $\begin{array}{l}\text { Força dos } \\
\text { relacionamentos }\end{array}$ & Fraca & Forte & $\begin{array}{l}\text { Em uma rede formada predominantemente } \\
\text { por joint ventures, o comprometimento } \\
\text { entre os parceiros é alto, tornando forte o } \\
\text { relacionamento entre os membros. }\end{array}$ \\
\hline $\begin{array}{l}\text { Experiência } \\
\text { com alianças } \\
\text { estratégicas }\end{array}$ & Pouca & Muita & $\begin{array}{l}\text { Considere que uma empresa tem muita } \\
\text { experiência, nesta situação, se ela possuir } \\
\text { alianças estratégicas por pelo menos } 10 \text { anos no } \\
\text { total, com pelo menos cinco parceiros distintos. }\end{array}$ \\
\hline $\begin{array}{l}\text { Volume de } \\
\text { investimentos } \\
\text { específicos }\end{array}$ & Baixo & Alto & $\begin{array}{l}\text { Um baixo volume de investimentos pode } \\
\text { existir em um acordo de prestação de } \\
\text { serviços, mas dificilmente ocorrerá em uma } \\
\text { joint venture. }\end{array}$ \\
\hline $\begin{array}{l}\text { Mecanismo de } \\
\text { governança }\end{array}$ & Inapropriado & Apropriado & $\begin{array}{l}\text { Se o custo do mecanismo de governança } \\
\text { for significativo, quando comparado ao custo } \\
\text { de cada transação entre as partes, então } \\
\text { o mecanismo de governança adotado é } \\
\text { inapropriado. }\end{array}$ \\
\hline $\begin{array}{l}\text { Grau de gestão } \\
\text { de mudanças na } \\
\text { rede }\end{array}$ & Baixo & Alto & $\begin{array}{l}\text { Quanto mais eficazes forem os processos de } \\
\text { gestão de mudanças na rede, maior será este } \\
\text { grau. }\end{array}$ \\
\hline $\begin{array}{l}\text { Grau de } \\
\text { alinhamento } \\
\text { dinâmico } \\
\text { múltiplo }\end{array}$ & Baixo & Alto & $\begin{array}{l}\text { Quanto mais avançados estiverem as } \\
\text { competências e processos que garantam } \\
\text { e sustentem a compatibilidade entre os } \\
\text { parceiros, maior será o grau de alinhamento } \\
\text { dinâmico múltiplo. }\end{array}$ \\
\hline
\end{tabular}

O questionário apresentava perguntas com vistas à definição do sinal de cada característica da RAE da empresa do respondente, apenas se ele estivesse trabalhando em uma empresa que possuísse alianças estratégicas. Este sinal era utilizado para contextualizar o cenário da resposta de cada influência, isto é, adaptar o questionário, tornando-o mais próximo da realidade do respondente. Por exemplo, se o respondente informasse que a cardinalidade era 
baixa na sua RAE, o questionário apresentaria o cenário "Considere a influência de uma rede com baixa cardinalidade sobre a TIC da organização"; caso contrário, ele apresentaria o cenário "Considere a influência de uma rede com alta cardinalidade sobre a TIC da organização".

Caso não fosse possível o respondente definir o sinal de alguma característica da RAE da sua empresa, pelo fato da informação não estar disponível ou por não ser de conhecimento do respondente, o questionário atribuía aleatoriamente um sinal, no momento da pergunta da influência daquela característica de RAE sobre as propriedades de TIC. Esse sinal, atribuído aleatoriamente, foi registrado junto com as respostas de cada questionário.

A influência RAE/TIC foi perguntada para cada característica de RAE, em relação a cada propriedade de TIC, e podia ser detalhada por elemento de TIC. Cada possível influência possui um sinal positivo (+), negativo (-) ou neutro (*). O conjunto de naturezas de influência é apresentado na tabela 1.

Tabela 1

Conjunto de naturezas de influências

\begin{tabular}{|lc|}
\hline Influência & Sinal \\
\hline Ativa & + \\
Desativa & - \\
Favorece & + \\
Desfavorece & - \\
Aumenta & + \\
Diminui & - \\
Facilita & + \\
Dificulta & - \\
Estimula & + \\
Inibe & - \\
Define & + \\
É definido por & - \\
Não afeta & $*$ \\
\hline
\end{tabular}

Assim, o respondente teve à sua disposição recursos para registrar com flexibilidade e detalhamento suas percepções de influência RAE/TIC. Por exemplo, ele pôde registrar que a centralidade (característica de RAE), considerando-se a influência da empresa focal ser central sobre a TIC da empresa focal (cenário), aumenta (influência) a complexidade da TIC da organização 
(propriedade de TIC), em especial da sua infra-estrutura e aplicações (elementos de TIC).

Como parte do questionário, foi construído um glossário com os principais conceitos de RAEs e TIC. Para cada característica de RAE foi apresentado um pequeno diagrama para melhor ilustrar o conceito. Consideramos fundamental o correto entendimento pelo respondente dos conceitos envolvidos, para não prejudicar a qualidade das respostas.

Para responder o questionário foram convidados 129 gestores de TI, dos quais 55 (43\%) responderam. Os respondentes demoraram em média 55 minutos para responder ao questionário.

Os dados coletados foram tratados estatisticamente quanto as suas tendências centrais e dispersão nos blocos B1 e B2, e principalmente por distribuição de freqüência nos blocos B3 e B4.

Por se tratar de uma amostra não-probabilística, os resultados não puderam ser analisados em termos da distribuição normal. No entanto, puderam ser objeto de uma generalização analítica. De fato, trouxeram subsídios relevantes para o planejamento estratégico e tomada de decisão dos gestores de TIC. Também trouxeram elementos significativos para consolidação do referencial teórico da pesquisa maior, da influência das RAEs sobre a TIC de uma empresa focal.

\section{Resultados}

Nas médias informadas nesta seção, sempre ao seu lado são apresentados a moda (mod), a mediana (med), o menor valor (min) e o maior valor (max) encontrados, entre parênteses.

\section{Respondente, empresas, alianças, RAE}

Os respondentes (bloco B1) possuíam em média idade de 47 anos ( $\bmod =46$; $\operatorname{med}=46 ; \min =34 ; \max =65)$; experiência profissional de 27 anos ( $\bmod =20$; $\operatorname{med}=25 ; \min =15 ; \max =45)$; experiência em TIC de 24 anos ( $\bmod =20$; $\operatorname{med}=23 ; \min =7 ; \max =40$ ); experiência em alianças estratégicas de nove anos ( $\bmod =10$; $\operatorname{med}=9 ; \min =0 ; \max =32)$; e 13 anos de experiência em gestão de TIC ( $\bmod =10 ; \operatorname{med}=10 ; \min =5 ; \max =32$ ).

Do total de respondentes, $76 \%$ caracterizaram a empresa onde trabalhavam (bloco B2). O tempo médio de existência destas empresas foi de 33 anos $(\bmod =50 ; \operatorname{med}=22 ; \min =1 ; \max =70)$. As distribuições de freqüência 
da quantidade de funcionários, área de atuação (segundo os setores utilizados pela edição Maiores e Melhores da revista Exame, Editora Abril) e faturamento estão nas tabelas 2,3 e 4, respectivamente.

Tabela 2

Número de funcionários

\begin{tabular}{|lc|}
\hline $\begin{array}{l}\text { Número de } \\
\text { funcionários }\end{array}$ & $\begin{array}{c}\text { Freqüência } \\
(\%)\end{array}$ \\
\hline Até 9 & 2,4 \\
De 10 a 19 & 2,4 \\
De 20 a 49 & 4,8 \\
De 50 a 99 & 0,0 \\
De 100 a 199 & 7,1 \\
De 200 a 499 & 14,3 \\
De 500 a 999 & 7,1 \\
De 1.000 a 1.999 & 9,5 \\
De 2.000 a 4.999 & 21,4 \\
Mais de 5.000 & 31,0 \\
\hline
\end{tabular}

Tabela 3

Áreas de atuação

\begin{tabular}{|lc|}
\hline Área & $\begin{array}{c}\text { Freqüência } \\
(\%)\end{array}$ \\
\hline Comércio varejista & 2 \\
Mineração & 2 \\
Serviços diversos & 2 \\
Automotivo & 2 \\
Serviços de transporte & 2 \\
Bancos & 5 \\
Farmacêutico, higiene e & 5 \\
cosméticos & \\
Comunicações & 5 \\
Telecomunicações & 5 \\
Química e petroquímica & 7 \\
Serviços públicos & 10 \\
Diversos & 10 \\
Seguradoras & 14 \\
Tecnologia e computação & 29 \\
\hline
\end{tabular}


Tabela 4

Faturamento

\begin{tabular}{|lc|}
\hline Faturamento & $\begin{array}{c}\text { Freqüência } \\
(\%)\end{array}$ \\
\hline Até $R \$ 1.200 .000$ (microempresa) & 2 \\
De $R \$ 1.201 .000$ até $R \$ 10.500 .000$ (pequena empresa) & 7 \\
De $R \$ 10.501 .000$ até $R \$ 60.000 .000$ (média empresa) & 12 \\
Acima de $R \$ 60.000 .000$ (grande empresa) & 67 \\
Informação não-disponível & 12 \\
\hline
\end{tabular}

Tabela 5

Tipos de alianças estratégicas

\begin{tabular}{|lc|}
\hline Tipo de & Freqüência \\
aliança & (\%) \\
\hline Acordo/Contrato de financiamento & 8 \\
Franquia & 8 \\
Licenciamento de patente ou know-how & 11 \\
Acordo/Contrato de P\&D & 18 \\
Investimento acionário minoritário & 18 \\
Participação acionária cruzada & 18 \\
Acordo/Contrato de produção & 18 \\
P\&D em conjunto & 24 \\
Acordo/Contrato de distribuição & 29 \\
Acordo/Contrato de comercialização & 29 \\
Joint venture & 32 \\
Transferência de tecnologia & 34 \\
Comercialização/Marketing em conjunto & 42 \\
Desenvolvimento/Co-produção & 45 \\
Acordo/Contrato de fornecimento & 50 \\
Acordo/Contrato de prestação de serviços & 71 \\
\hline
\end{tabular}

A caracterização das alianças estratégicas e da RAE da empresa onde trabalha (bloco B3) foi realizada por 90\% do total de respondentes. Essas empresas possuíam em média 16 alianças estratégicas $(\bmod =3 ; \operatorname{med}=9 ; \min =1$; $\max =90)$ e experiência de 10 anos $(\bmod =10 ; \operatorname{med}=10 ; \min =1 ; \max =50)$ com essas alianças. A distribuição de freqüência dos tipos de alianças estratégicas encontra-se na tabela 5.

Na caracterização da RAE da empresa onde trabalha, em 29\% dos casos, o respondente atribuiu às características um valor com sinal negativo (baixo, fraco, pouco etc.), em $62 \%$ dos casos, às características foi atribuído um valor 
com sinal positivo (alto, forte, muito etc.) e em $9 \%$ dos casos a informação não esteve disponível. Ao final da atribuição aleatória de sinais aos casos sem sinal, foram apresentados para os respondentes 39\% de cenários com sinal negativo e $61 \%$ com sinal positivo.

\section{Influências sobre TIC}

As tabelas 6 a 9 apresentam sinteticamente os resultados das influências das características de RAE sobre as propriedades de TIC. No conjunto de colunas "cenário +" são apresentadas as freqüências das respostas para o cenário positivo (ex.: "Considere uma RAE com alta centralidade"). A primeira coluna desse conjunto mostra o percentual de influências de sinal positivo que foram assinaladas para cada característica de RAE, a segunda mostra a neutralidade da influência e a última o percentual de influências de sinal negativo que foram assinaladas. O mesmo ocorre para o conjunto de colunas "cenário -" (cenário negativo).

Os comentários dos resultados, por propriedade de TIC, consideram aspectos de simetria-assimetria, força e indefinição nas percepções de influência. Não há, inerentemente à natureza do fenômeno, expectativas de simetria ou de assimetria para as influências.

Consideramos que há "simetria positiva" quando, no cenário positivo, a característica de RAE influencia positivamente a propriedade e, ao mesmo tempo, ele influencia negativamente a mesma propriedade no cenário negativo. Por exemplo, há simetria positiva na influência do mecanismo de governança (Mecgov) sobre a complexidade (vide tabela 8), ou seja, em um cenário de mecanismos de governança adequados, maior será a complexidade de TIC, e em um cenário de mecanismos de governança inadequados, menor será essa mesma complexidade. Características de RAE com simetria positiva têm influência positiva quando presentes, e negativa quando ausentes. Da mesma forma, existe "simetria negativa", como na influência do alinhamento dinâmico múltiplo (ADM) sobre a complexidade (na tabela 7).

Há "assimetria positiva" quando, no cenário positivo, a característica de RAE influencia positivamente a propriedade e, ao mesmo tempo, influencia positivamente essa mesma propriedade no cenário negativo. Por exemplo, há assimetria positiva na influência da cardinalidade sobre a maturidade (tabela 6), ou seja, em um cenário de alta cardinalidade, maior será a maturidade de TIC, e também em um cenário de baixa cardinalidade, maior será essa mesma maturidade. Características de RAE com assimetria positiva sempre têm 
influência positiva, ou seja, basta a empresa participar de uma RAE que ela é afetada positivamente por essa característica. Analogamente, poderia existir uma "assimetria negativa" (nenhum caso foi identificado).

Para o aspecto de força da influência consideramos que ela é forte quando $70 \%$ ou mais dos respondentes apontaram aquele sinal para a influência. Assim, a influência do grau de gestão de mudanças na rede (GGMR) sobre a maturidade de TIC foi considerada de "assimetria forte com efeito positivo" (tabela 6). Há situações que em um cenário há influência e no outro não há definição nas percepções. Os cenários considerados indefinidos foram os em que a diferença entre as duas maiores freqüências não foi superior a 14\%. Por exemplo, a influência do status da empresa focal (Stafoc) sobre a complexidade de TIC (tabela 7) apresenta influência positiva no cenário positivo e não apresenta influência definida no cenário negativo. Nesses casos consideramos que há influência parcial com efeito positivo. Por fim, há indefinição nas percepções quando não há indicação clara de simetria ou assimetria, e nenhuma influência é forte. Por exemplo, há indefinição na influência do status dos parceiros (Stapar) sobre o alinhamento de TIC (tabela 9).

$$
\text { Tabela } 6
$$

Influências sobre a maturidade de TIC (\%)

\begin{tabular}{|lrrrrrrrr|}
\hline \multirow{2}{*}{ RAE } & \multicolumn{3}{c}{ Cenário +} & & \multicolumn{3}{c|}{ Cenário - } \\
\cline { 2 - 3 } \cline { 7 - 8 } & + & $*$ & - & & + & $*$ & - \\
\hline CAR & 86 & 9 & 5 & & 60 & 10 & 30 \\
Dens & 80 & 3 & 17 & & 60 & 20 & 20 \\
Centr & 80 & 10 & 10 & & 63 & 24 & 13 \\
Equiv & 73 & 11 & 16 & & 67 & 22 & 11 \\
Stafoc & 94 & 3 & 3 & & 32 & 15 & 53 \\
Stapar & 73 & 11 & 16 & & 39 & 33 & 28 \\
Natrel & 88 & 7 & 5 & & 21 & 15 & 64 \\
Forrel & 87 & 3 & 10 & & 44 & 16 & 40 \\
Expae & 90 & 7 & 3 & & 31 & 19 & 50 \\
Volie & 77 & 10 & 13 & & 16 & 28 & 56 \\
Mecgov & 85 & 5 & 10 & & 20 & 7 & 73 \\
GGMR & 88 & 0 & 12 & & 19 & 5 & 76 \\
ADM & 92 & 0 & 8 & & 33 & 10 & 57 \\
\hline
\end{tabular}

Cardinalidade (CAR); densidade (Dens); centralidade (Centr); equivalência estrutural (Equiv); status da empresa focal (Stafoc); status dos parceiros (Stapar); natureza do relacionamento (Natrel); força do relacionamento (Forrel); experiência com alianças estratégicas (Expae); volume de investimentos específicos (Volie); mecanismo de governança (Mecgov); grau de gestão de mudanças na rede (GGMR); alinhamento dinâmico múltiplo (ADM). 
Tabela 7

Influências sobre a complexidade de TIC (\%)

\begin{tabular}{|lrrrrrrrr}
\hline \multirow{2}{*}{ RAE } & \multicolumn{3}{c}{ Cenário +} & & \multicolumn{3}{c|}{ Cenário - } \\
\cline { 2 - 3 } \cline { 8 - 9 } & + & $*$ & - & & + & $*$ & - \\
\hline CAR & 91 & 0 & 9 & & 45 & 5 & 50 \\
Dens & 90 & 0 & 10 & & 52 & 16 & 32 \\
Centr & 61 & 4 & 35 & & 63 & 12 & 25 \\
Equiv & 46 & 19 & 35 & & 56 & 11 & 33 \\
Stafoc & 75 & 3 & 22 & & 37 & 26 & 37 \\
Stapar & 65 & 21 & 14 & & 50 & 33 & 17 \\
Natrel & 29 & 27 & 44 & & 58 & 21 & 21 \\
Forrel & 40 & 10 & 50 & & 44 & 28 & 28 \\
Expae & 48 & 14 & 38 & & 65 & 20 & 15 \\
Volie & 57 & 20 & 23 & & 44 & 28 & 28 \\
Mecgov & 45 & 12 & 43 & & 60 & 7 & 33 \\
GGMR & 53 & 9 & 38 & & 48 & 9 & 43 \\
ADM & 32 & 8 & 60 & & 70 & 10 & 20 \\
\hline
\end{tabular}

Cardinalidade (CAR); densidade (Dens); centralidade (Centr); equivalência estrutural (Equiv); status da empresa focal (Stafoc); status dos parceiros (Stapar); natureza do relacionamento (Natrel); força do relacionamento (Forrel); experiência com alianças estratégicas (Expae); volume de investimentos específicos (Volie); mecanismo de governança (Mecgov); grau de gestão de mudanças na rede (GGMR); alinhamento dinâmico múltiplo (ADM).

$$
\text { Tabela } 8
$$

Influências sobre a flexibilidade de TIC (\%)

\begin{tabular}{|lrrrrrrrr|}
\hline \multirow{2}{*}{ RAE } & \multicolumn{3}{c}{ Cenário +} & & \multicolumn{3}{c|}{ Cenário - } \\
\cline { 2 - 4 } \cline { 7 - 8 } & + & $*$ & - & & $*$ & - \\
\hline CAR & 74 & 3 & 23 & & 50 & 5 & 45 \\
Dens & 50 & 3 & 47 & 36 & 20 & 44 \\
Centr & 68 & 3 & 29 & 58 & 4 & 38 \\
Equiv & 46 & 19 & 35 & 61 & 0 & 39 \\
Stafoc & 42 & 16 & 42 & 32 & 21 & 47 \\
Stapar & 32 & 22 & 46 & 50 & 33 & 17 \\
Natrel & 75 & 15 & 10 & 29 & 21 & 50 \\
\hline
\end{tabular}




\begin{tabular}{|lrrrrrrrr|}
\hline \multirow{2}{*}{ RAE } & \multicolumn{3}{c}{ Cenário +} & & \multicolumn{3}{c|}{ Cenário - } \\
\cline { 2 - 3 } \cline { 8 - 9 } & + & $*$ & - & & + & $*$ & - \\
\hline Forrel & 63 & 10 & 27 & & 40 & 16 & 44 \\
Expae & 76 & 14 & 10 & & 30 & 12 & 58 \\
Volie & 70 & 7 & 23 & & 28 & 24 & 48 \\
Mecgov & 60 & 12 & 28 & & 0 & 7 & 93 \\
GGMR & 71 & 8 & 21 & & 14 & 19 & 67 \\
ADM & 72 & 4 & 24 & & 23 & 10 & 67 \\
\hline
\end{tabular}

Cardinalidade (CAR); densidade (Dens); centralidade (Centr); equivalência estrutural (Equiv); status da empresa focal (Stafoc); status dos parceiros (Stapar); natureza do relacionamento (Natrel); força do relacionamento (Forrel); experiência com alianças estratégicas (Expae); volume de investimentos específicos (Volie); mecanismo de governança (Mecgov); grau de gestão de mudanças na rede (GGMR); alinhamento dinâmico múltiplo (ADM).

Tabela 9

Influências sobre o alinhamento de TIC (\%)

\begin{tabular}{|c|c|c|c|c|c|c|}
\hline \multirow{2}{*}{ RAE } & \multicolumn{3}{|c|}{ Cenário + } & \multicolumn{3}{|c|}{ Cenário - } \\
\hline & + & $*$ & - & + & $*$ & - \\
\hline CAR & 66 & 8 & 26 & 55 & 15 & 30 \\
\hline Dens & 50 & 13 & 37 & 52 & 16 & 32 \\
\hline Centr & 77 & 13 & 10 & 63 & 12 & 25 \\
\hline Equiv & 56 & 22 & 22 & 44 & 23 & 33 \\
\hline Stafoc & 75 & 11 & 14 & 26 & 27 & 47 \\
\hline Stapar & 54 & 8 & 38 & 33 & 34 & 33 \\
\hline Natrel & 88 & 7 & 5 & 43 & 14 & 43 \\
\hline Forrel & 83 & 7 & 10 & 40 & 12 & 48 \\
\hline Expae & 90 & 7 & 3 & 35 & 19 & 46 \\
\hline Volie & 80 & 7 & 13 & 20 & 32 & 48 \\
\hline Mecgov & 80 & 10 & 10 & 13 & 13 & 74 \\
\hline GGMR & 82 & 9 & 9 & 10 & 10 & 81 \\
\hline ADM & 88 & 0 & 12 & 20 & 17 & 63 \\
\hline
\end{tabular}

Cardinalidade (CAR); densidade (Dens); centralidade (Centr); equivalência estrutural (Equiv); status da empresa focal (Stafoc); status dos parceiros (Stapar); natureza do relacionamento (Natrel); força do relacionamento (Forrel); experiência com alianças estratégicas (Expae); volume de investimentos específicos (Volie); mecanismo de governança (Mecgov); grau de gestão de mudanças na rede (GGMR); alinhamento dinâmico múltiplo (ADM). 


\section{Influências sobre os elementos de TIC}

Para os respondentes, em $86 \%$ dos casos todos os elementos de TIC (infra-estrutura, aplicações, processos, equipe e gestão) são influenciados. A infraestrutura foi citada em 93\%; as aplicações em 94\%; os processos em 96\%; a equipe em $92 \%$; e a gestão em $94 \%$ das influências.

\section{Resumo das influências sobre TIC}

A tabela 10 apresenta um resumo dos aspectos de simetria-assimetria, força e indefinição nas percepções de influência das características de RAE sobre as propriedades de TIC.

Tabela 10

Resumo das influências sobre a TIC

\begin{tabular}{|lcccc|}
\hline Discriminação & Maturidade & Complexidade & Flexibilidade & Alinhamento \\
\hline Cardinalidade & ASt & AN+ & AN+ & AS+ \\
Densidade & AS+ & AS+ & IN & IN \\
Centralidade & AS+ & AS+ & AS+ & AS+ \\
Equivalência estrutural & AS+ & IN & IN & IN \\
Status da empresa focal & S+ & AN+ & IN & S+ \\
Status dos parceiros & AN+ & AS+ & IN & IN \\
Natureza do relacionamento & S+ & S- & S+ & AN+ \\
Força do relacionamento & AN+ & IN & IN & AN+ \\
Experiência com alianças estratégicas & S+ & IN & S+ & AN+ \\
Volume de investimentos específicos & S+ & AS+ & S+ & S+ \\
Mecanismo de governança & SF+ & IN & S+ & SF+ \\
Grau de gestão de mudanças na rede & SF+ & IN & S+ & SF+ \\
Alinhamento dinâmico múltiplo & S+ & S- & S+ & S+ \\
\hline
\end{tabular}

Simetria forte com efeito positivo (SF+); simetria com efeito positivo (S+); simetria com efeito negativo (S-); assimetria forte com efeito positivo (ASF+); assimetria com efeito positivo (AS+); parcial com efeito positivo no cenário positivo $(\mathrm{AN}+)$; parcial com efeito positivo no cenário negativo $(\mathrm{BN}+)$; indefinido (IN).

Das 52 possíveis influências (13 características de RAE, quatro propriedades de TIC), 20 exibem sinais de simetria (18 com efeito positivo e duas com efeito negativo), 11 exibem sinais de assimetria (todas com efeito positivo), 
oito apresentam efeito parcialmente positivo e as restantes 13 são indefinidas. Não foram identificadas assimetrias negativas.

Quanto à força, apenas quatro foram consideradas fortes (70\% ou mais dos respondentes apontaram aquele sinal para a influência). Observando-se apenas as influências fortes, tem-se que as propriedades de TIC mais influenciadas foram a maturidade e o alinhamento. Não foi identificada nenhuma característica que tivesse influência forte sobre as quatro propriedades de TIC investigadas.

Considerando-se que as influências parciais e as indefinições são menos relevantes que as demais, os dados sugerem que a equivalência estrutural é a característica de RAE que menos influencia as propriedades de TIC da empresa focal. As características de RAE mais influentes, aqui definidas como as que apresentaram pelo menos uma influência forte, foram o mecanismo de governança e o grau de gestão de mudanças na rede. As propriedades de TIC com maior indefinição, quanto à influência da RAE, foram a complexidade e a flexibilidade.

\section{Discussão}

A pesquisa trouxe fortes indícios de que a influência das características de RAEs sobre a TIC da empresa focal existe e se manifesta de várias formas e com distintos graus de força.

A área de TIC precisa estar atenta à inserção da empresa em RAEs. Consideremos que a empresa inicialmente participa de uma "RAE mínima", onde todas as suas características da RAE têm sinal negativo (baixa cardinalidade, a empresa focal não é central, poucos investimentos etc.) e que eventualmente essa mesma RAE se torna uma "RAE máxima" com todas as suas características com sinal positivo (alta cardinalidade, a empresa focal é central, muitos investimentos etc.).

Quando essa empresa, que não era membro de nenhuma RAE, começa a participar de uma "RAE mínima", a sua área de TIC fica exposta às influências assimétricas da RAE. As assimetrias positivas sugerem que a presença da RAE, independentemente do cenário (positivo ou negativo) das suas características, influencia positivamente as propriedades de TIC da empresa focal. Nesse caso, o gestor de TIC precisa se antecipar e ter a sua área preparada para ter maiores maturidade e complexidade. Essas são conseqüências imediatas da participação da empresa em qualquer RAE.

Com a mudança das características da RAE da empresa, por hipótese, em direção à "RAE máxima", novos efeitos podem ser esperados sobre as 
propriedades da área de TIC, devido às influências simétricas desta RAE. O gestor de TIC deve ter especial atenção ao mecanismo de governança e grau de gestão de mudanças na rede, pois são características de RAE que exercem influências simétricas e fortes. As propriedades de TIC mais afetadas pelas influências simétricas são a maturidade e a flexibilidade.

A tabela 10, que apresenta o resumo da influência de cada característica de RAE sobre cada propriedade de TIC, pode ser usado como apoio ao gestor de TIC, na sua avaliação de quais propriedades de TIC necessitam de maior atenção, à medida que a empresa começa a participar de uma RAE e a rede evolui.

Por exemplo, se a RAE da empresa é uma "RAE mínima" e a sua cardinalidade aumenta, o gestor de TIC deve dar atenção aos possíveis efeitos sobre a complexidade de TIC, pois mais parceiros estratégicos podem trazer novas necessidades, obrigando a empresa focal a ter mais variedade em TIC. Se, após esta primeira mudança, os mecanismos de governança desta RAE se tornarem apropriados, e conseqüentemente o conjunto de salvaguardas dos relacionamentos entre as partes para evitar que nenhuma delas perceba benefícios em comportamentos oportunistas evoluírem, o gestor de TIC deve ficar atento às propriedades maturidade e alinhamento da sua área de TIC.

Uma possível interpretação para o fato da maturidade e do alinhamento serem as propriedades de TIC mais influenciadas pelas características de uma RAE é que a participação da empresa focal em uma RAE obriga a sua TIC a tornar-se mais madura (mais evoluída ou com mais competência) pois ela passa a se relacionar com as áreas de TIC de várias empresas, e necessariamente mais alinhada internamente (coerente com a estratégia de negócios), de modo a estar apta a melhor resolver eventuais conflitos, com um maior número de parceiros estratégicos.

Surpreendentemente, de acordo com os resultados, a flexibilidade de TIC não é afetada por influências fortes da RAE. No entanto, ao analisarmos com mais atenção esse ponto, ficou claro que a flexibilidade não era percebida como tão crítica, pois há uma tendência de elementos da TIC se tornarem commodities (Carr, 2003), em especial a sua infra-estrutura (Broadbent e Weill, 1997). Esse fato faz com que a necessidade de flexibilidade de TIC diminua, pois caso sejam necessários novos equipamentos, estes podem ser rapidamente obtidos no mercado. Por outro lado, do ponto de vista estratégico, a flexibilidade de TIC está atrelada à flexibilidade dos processos de negócio. A necessidade de flexibilidade em TIC provavelmente é mais determinada pela necessidade de flexibilidade dos processos de negócio do que pela sua participação em uma RAE. 


\section{Conclusão}

Neste artigo apresentamos os resultados mais relevantes de uma investigação empírica exploratória das percepções de gestores de TIC — tecnologia de informação e comunicação, conduzida no Brasil pelos autores, relativas às influências exercidas sobre quatro propriedades de TIC (maturidade, complexidade, flexibilidade e alinhamento) de uma empresa focal pela sua RAE — rede de alianças estratégicas.

Outras pesquisas já demonstraram a importância da análise das características das RAEs para o planejamento estratégico de uma empresa (Macedo-Soares e Lange, 2004; Leite e Macedo-Soares, 2006), porém, conforme mencionado, este é o primeiro estudo que confirma e detalha essas influências no que diz respeito à TIC de uma empresa.

De fato, os resultados sugerem fortemente que, também no caso de planejamento estratégico de TIC, não podemos deixar de considerar o impacto da RAE da qual a empresa participa, sob pena de: ser ineficiente, pela priorização inadequada para alocação de recursos e maiores gastos; e ser ineficaz, por não atender às necessidades e condições impostas pela RAE da empresa.

O resumo de influências apresentado neste artigo pode auxiliar o gestor de TIC de uma empresa a priorizar suas ações sobre as propriedades de TIC, em função da participação da empresa em uma RAE e das características desta. Ao reconhecerem essas influências, e tendo em vista que as características da RAE da sua empresa estão em mudança contínua, os gestores de TIC podem antecipar-se às necessidades das suas empresas garantindo, portanto, a coerência da TIC com a estratégia da empresa de modo a acompanhar suas necessidades (alinhamento estratégico).

Entre os gestores de TIC há uma dificuldade de levar em conta as características da RAE da empresa e suas influências sobre o planejamento estratégico da área. Os resultados da pesquisa trouxeram subsídios para diferenciar quais as características mais relevantes. Por exemplo, há impacto imediato sobre a TIC da empresa quando ela começa a participar de uma RAE, o que é comprovado pelas influências assimétricas identificadas, em especial as que afetam a maturidade. A complexidade de TIC também sofre influências assimétricas da RAE, porém em maior quantidade são as suas influências indefinidas. À medida que a RAE evolui, o foco de atenção do gestor de TIC deve estar voltado para maturidade e flexibilidade da sua área. As características de RAE relacionadas à administração da rede (Macedo-Soares, 2002) são as que mais influenciam o alinhamento da área de TIC. 
Por fim, conforme foi destacado, a investigação foi exploratória. Seus resultados trouxeram subsídios significativos e fundamentais para as próximas etapas da pesquisa maior na qual esta se insere. Recomendamos que, em paralelo, outros pesquisadores também contribuam para uma gestão estratégica de TIC mais eficiente e eficaz no caso de empresas que atuem em RAEs, focando em setores específicos (ex.: bancos) e tipos de alianças estratégicas (ex.: joint ventures).

\section{Referências bibliográficas}

ANDERSEN, T. J. Information technology, strategic decision making approaches and organizational performance in different industrial settings. Journal of Strategic Information Systems, v. 10, n. 2, p. 101-119, June 2001.

BROADBENT, M.; WEILL, P. Management by Maxim: how business and IT managers can create IT infrastructures. Sloan Management Review, v. 38, n. 3, p. 77-92, Spring 1997.

BYRD, T. A. Information technology: core competencies, and sustained competitive advantage. Information Resource Management Journal, v. 14, n. 2, p. 27-36, Apr./June 2001.

CARR, N.G. It doesn't matter. Harvard Business Review, v. 81, n. 5, May 2003.

DEWETT, T.; JONES, G. R. The role of information technology in the organization: a review, model, and assessment. Journal of Management, v. 27, n. 3, p. 313-346, May 2001.

DUIMERING, P. R.; SAFAYENI, F; PURDY, L. Integrated manufacturing: redesign the organization before implementing flexible technology. Sloan Management Review, v. 34, n. 4, p. 47-56, Summer 1993.

DYER, J. H.; SINGH, H. The relational view: cooperative strategy and sources of interorganizational competitive advantage. The Academy of Management Review, v. 23, n. 4, p. 660-679, Oct. 1998.

GALASKIEWICZ, J.; ZAHEER, A. Networks of competitive advantage. Research in the Sociology of Organizations, v. 16, p. 237-261, 1999.

GULATI, R. Alliances and networks. Strategic Management Journal, v. 19, n. 4, p. 293-317, 1998.

; NOHRIA, N.; ZAHEER, A. Strategic networks. Strategic Management Journal, v. 21, n. 3, p. 203-215, Mar. 2000. 
HAMMER, M.; CHAMPY, J. Reengineering the corporation: a manifesto for business revolution. New York: HarperBusiness, 1995.

HENDERSON, J. C.; VENKATRAMAN, N. Strategic alignment: leveraging information technology for transforming organizations. IBM Systems Journal, v. 32, n. 1, p. 4-16, 1993.

ITGI. COBIT 4.1 - Control objectives for information technology, IT Governance Institute, Rolling Meadows, 2007.

KALE, P.; SINGH H.; PERLMUTTER, H. Learning and protection of proprietary assets in strategic alliances: building relational capital. Strategic Management Journal, v. 21, n. 3, p. 217-237, Mar. 2000.

KNOKE, D. Changing organizations: business networks in the new political economy. Boulder, CO: Westview, 2001.

KNOLL, K., JARVENPAA, S. L. Information technology alignment or "fit" in highly turbulent environments: the concept of flexibility. In: COMPUTER PERSONNEL RESEARCH CONFERENCE ON REINVENTING IS: MANAGING INFORMATION TECHNOLOGY IN CHANGING ORGANIZATIONS. Proceedings...Virginia, United States, 1994.

KONSYNSKI, B. R. Strategic control in the extended enterprise. IBM Systems Journal, v. 32, n. 1, p. 111-142, 1993.

LEITE, J. C.; MACEDO-SOARES, T. D. L. v. A. Alianças e redes estratégicas no setor de downstream de petróleo no Brasil. Revista Brasileira de Administração Pública, v. 39, n. 6, p. 1319-1347, 2006.

LIA, J. et al. A strategic analysis of inter organizational information sharing. Decision Support Systems, v. 42, n. 1, p. 251-266, 2006.

LUFTMAN, J. N.; PAPP, R.; BRIER, T. Enablers and inhibitors of business-IT alignment. Communications of AIS, v. 1, n. 11, Mar. 1999.

MACEDO-SOARES, T. D. L. v. A. Strategic alliances and networks: conceptual tools for strategic assessments. Readings Book of GBATA International Conference. Rome: St. John's University, 2002. p. 292-305.

; LANGE, T. T. Ferramental para análise estratégica pela ótica relacional: resultados do seu teste piloto na Companhia Vale do Rio Doce (CVRD). In: ENANPAD, 2002. Salvador, Bahia. Anais... ESO 1525, Rio de Janeiro, 2002.

MARCHAND, D. A.; KETTINGER, W. J.; ROLLINS, J. D. Information orientation: people, technology and the bottom line. MIT Sloan Management Review, v. 41, n. 4, p. 69-80, 2000. 
McFARLAN, F. W. Information technology changes the way you compete. Harvard Business Review, v. 62, n. 3, p. 98-103, May/June 1984.

. Information partnerships - shared data, shared scale. Harvard Business Review, v. 68, n. 5, p. 114-120, Sept./Oct. 1990.

PORTER, M. How competitive forces shape strategy. Harvard Business Review, v. 57, n. 2, p. 137-145, Mar./Apr. 1979.

PRAHALAD, C. K.; HAMEL, G. The core competence of the corporation. Harvard Business Review, v. 68, n. 3, p. 79-81, May/June 1990.

; KRISHNAN, M. S. The dynamic synchronization of strategy and information technology. MIT Sloan Management Review, v. 43, n. 4, p. 24-33, 2002.

REA, L. M.; PARKER, R. A. Metodologia de pesquisa: do planejamento à execução. Pioneira, 2000.

REMENYI, D. et al. Doing research in business and management: an introduction to process and method. London: Sage, 1998.

ROCKART, J. F; EARL, M. J.; ROSS, J. W. Eight imperatives for the new IT organization. Sloan Management Review, v. 38, n. 1, p. 43-55, 1996.

. Towards survivability of communication-intensive new organization forms. Journal of Management Studies, v. 35, n. 4, p. 417-420, July 1998.

RUSSO, G. M.; VILLAS, M.; MACEDO-SOARES, T. D. L. v. A. Importância da hierarquização das revistas científicas: resultados de uma investigação empírica no Brasil e proposta de um método de pesquisa bibliográfica. In: ENANPAD 2006, Salvador, Bahia. Anais... Rio de Janeiro, Anpad, 2006.

SAMPLER, J. L.; SHORT, J. E. Strategy in dynamic information-intensive environments. Journal of Management Studies, v. 35, n. 4, p. 429-436, July 1998.

SILVA, A. L. da; ALCÂNTARA, R. C. Mudanças nos relacionamentos e estratégias para melhor coordenação da cadeia de suprimentos. Revista de Administração da Universidade de São Paulo, v. 36, n. 4, p. 49-58, jul./set. 2001.

SHAPIRO, C.; VARIAN, H. R. The art of standards wars. California Management Review, v. 41, n. 2, 1999.

VILLAS, M. V.; MACEDO-SOARES, T. D. L. v. A.; FONSECA, M. V. M. Assegurando alinhamento estratégico da tecnologia de informação e comunicação: o caso das unidades de refino da Petrobras. Revista de Administração Pública, v. 40, n. 1, p. 127-53, jan./fev. 2006. 